\title{
HUSEMOLLER-WITT DECOMPOSITIONS AND ACTIONS OF THE STEENROD ALGEBRA
}

\author{
by ANDREW BAKER
}

(Received 9th August 1984)

(To the memory of my Mother)

\section{Introduction}

Recently, there has been renewed interest in the homology of connective covers of the classifying spaces $B U$ and $B O$, and their associated Thom spectra-see e.g. $[4,6,9,10,15]$. There are now numerous families of generators as well as structural results on the action of the Steenrod algebra. However, these two areas have not been well related since the methods used have tended to emphasise one goal rather than the other. In this paper we show that there are in fact canonical Hopf algebra decompositions for the sub-Hopf algebras of the homology of $B U$, and $B O$ constructed by $\mathrm{S}$. Kochman in [9], generalising those of [8]. Furthermore, these are clearly and consistently related to the Steenrod algebra action, and provide canonical sets of algebra generators. They should thus allow calculations of the type exemplified in [6] to be carried out in all cases, although of course the complexity of the answer increases rapidly! A by-product of our approach is that we can easily obtain results on these homologies as Hopf algebras, such as self-duality and a computation of endomorphism groups over the Steenrod algebra. We feel that the methods will also give interesting information in the case of some other familiar spaces even if their homology is not self dual (or bipolynomial); we intend to return to this in a sequel.

The main results of this paper are the following. Theorems (1.11), (1.13) contain the Hopf algebra decompositions of Kochman's algebras; (2.10) and (2.11) describe how to calculate the (co)action of the (dual) Steenrod algebra on the homology algebras; Theorem (3.1) describes the images of Kochman's algebras under the relevant Thom isomorphisms; Theorem (4.3) which calculates all endomorphisms of these Hopf algebras which commute with Steenrod operations.

I wish to thank the SERC for support whilst this work was completed, and the following for correspondence and stimulation: David Pengelley, Vince Giambalvo, Stan Kochman, Peter Hoffman, Arunas Liulevicius, Peter May and Clarence Wilkerson.

1.

Throughout, let $p$ be a fixed prime. Then after localisation at $p, B U$ splits as a product (of infinite loop spaces)

$$
\prod_{0 \leqq i \leqq p-2} B U_{p, i}[1] .
$$

We will denote $B U_{p, 0}$ by $W$, with cohomology (for a $p$-local ring $R$ )

$$
H^{*}(W ; R)=R\left[v_{i}: i \geqq 1\right] \subset H^{*}(B U ; R)
$$


where $\left|v_{i}\right|=2(p-1) i$. We can actually choose $v_{i}$ so that the (Whitney sum) diagonal is

$$
\Delta\left(v_{i}\right)=\sum_{0 \leqq j \leqq i} v_{j} \otimes v_{i-j}
$$

Let $s_{k} \in H^{2 k}(B U ; R)$ denote the standard primitive generator, given as the kth Newton polynomial in the Chern classes $c_{i}$. Then $s_{(p-1) i} \in H^{*}(W ; R)$. The Husemoller-Witt decomposition of $[8]$ induces an $R$-Hopf algebra splitting

$$
H^{*}(W ; R)=\bigotimes_{(k, p)=1}(B[2 k(p-1)] \otimes R)
$$

where

$$
B[2 k]=\mathbb{Z}_{(p)}\left[a_{k, j} ; j \geqq 0\right]
$$

and $\left|a_{k, j}\right|=2 k p^{j}$; we always assume $(k, p)=1$ when discussing such Hopf algebras. The coproduct on $B[2 k]$ is such that the primitives $P B[2 k]$ are generated by elements $q_{k p j}$ of degree $2 k p^{j}$ with

$$
q_{k p^{j}}=p^{j} a_{k, j}+p^{j-1} a_{k, j-1}^{p}+\cdots+p a_{k, 1}^{p^{j-1}}+a_{k, 0}^{p^{j}}
$$

The coproduct on the $a_{k, j}$ then takes the form

$$
\Delta\left(a_{k, j}\right)=\Sigma_{j}^{W}\left(a_{k, 0} \otimes 1, \ldots, a_{k, j} \otimes 1 ; 1 \otimes a_{k, 0}, \ldots, 1 \otimes a_{k, j}\right)
$$

where $\Sigma_{j}^{W}\left(X_{0}, \ldots, X_{j} ; \quad Y_{0}, \ldots, Y_{j}\right) \in \mathbb{Z}_{(p)}\left[X_{0}, \ldots, X_{j}, Y_{0}, \ldots, Y_{j}\right]$ is the $j$ th Witt addition polynomial (see for example [7]). $\Sigma_{j}^{W}$ can be computed recursively from the fact that $q_{k p j}$ is primitive.

Each $B[2 k]$ is a self dual, bipolynomial, indecomposable Hopf algebra, possessing several remarkable properties, some of which are discussed in [16]. We will make use of two structure maps: the Frobenius $F: B[2 k] \rightarrow B[2 k]$, and the Verschiebung $V: B[2 k] \rightarrow B[2 k]$.

$F$ multiplies degrees by $p$, and $V$ divides degrees by $p$. To define $F, V$ we first introduce elements $a_{k, j}^{(1)} \in B[2 k]$ of degree $2 k p^{j+1}$ and satisfying (see [3])

$$
q_{k p^{j+1}}=p^{j} a_{k, j}^{(1)}+p^{j-1}\left(a_{k, j-1}^{(1)}\right)^{p}+\cdots+p\left(a_{k, 1}^{(1)}\right)^{p^{j-1}}+\left(a_{k, 0}^{(1)}\right)^{p^{j}} .
$$

Then we define

$$
\begin{array}{rlrl}
F\left(a_{k, j}\right) & =a_{k, j}^{(1)} & & \\
V\left(a_{k, j}\right) & =0, & j=0 \\
& =a_{k, j-1}, & j>0 .
\end{array}
$$

These are both Hopf algebra endomorphisms (in the non-grade preserving sense) 
satisfying as basic relations

$$
F V=V F=[p]
$$

where $[p]$ denotes the $p$-fold sum in the abelian group of Hopf algebra endomorphisms of $B[2 k]$. Note also that upon dualising and making the identification

$$
B[2 k] \cong \operatorname{Hom}_{(p)}\left(B[2 k] ; \mathbb{Z}_{(p)}\right)
$$

the adjoints of $F$ and $V$ are identified with $V$ and $F$ respectively.

We will make use of the elements $a_{k, j}^{(r)}$ defined by

$$
a_{k, j}^{(r)}=F^{r}\left(a_{k, j}\right)=F\left(a_{k, j}^{(r-1)}\right)
$$

We define

$$
\begin{aligned}
B^{(r)}[2 k] & =\operatorname{im} F^{r}: B[2 k] \rightarrow B[2 k] \\
& =\mathbb{Z}_{(p)}\left[a_{k, j}^{(r)}: j \geqq 0\right]
\end{aligned}
$$

which is a sub-Hopf algebra of $B[2 k]$, with $F^{r}$ an isomorphism of Hopf algebras onto $B^{(r)}[2 k]$. Note that from the self duality map for $B[2 k]$ we obtain an isomorphism

$$
B[2 k] / / \mathbb{Z}_{(p)}\left[a_{k, j}: 0 \leqq j<r\right] \cong \operatorname{Hom}_{\mathbf{Z}_{(p)}}\left(B^{(r)}[2 k] ; \mathbb{Z}_{(p)}\right) .
$$

We also have (see [3])

$$
\begin{gathered}
q_{k p^{j+r}}=p^{j} a_{k, j}^{(r)}+p^{j-1}\left(a_{k, j-1}^{(r)}\right)^{p}+\cdots+p\left(a_{k, 1}^{(r)}\right)^{p^{j-1}}+\left(a_{k, 0}^{(r)}\right)^{p^{j}} \\
a_{k, j}^{(r)} \equiv p^{r} a_{k, j+r} \quad(\bmod \text { decomposables }) \\
a_{k, j}^{(r)} \equiv a_{k, j}^{p^{\prime}} \quad(\bmod p)
\end{gathered}
$$

(both relations in $B[2 k])$.

From now on, let $\bar{B}[2 k]=B[2 k] \otimes \mathbb{F}_{p}$.

We next describe the action of the Steenrod pth powers in $H^{*}(B U)=H^{*}\left(B U ; \mathbb{F}_{p}\right)$. Recall that for each $s \geqq 0$ there is an additive operation $\mathscr{P}^{s}$, raising degree by $2(p-1) s$. We denote by $\mathscr{P}^{*}$ the Hopf algebra generated by these, subject to the Adem relations. Note that if $p=2, \mathscr{P}^{s}=S q^{2 s}$. (We will discuss the analogous situation for $H^{*}(B O)$ later). Of course $\mathscr{P}^{*}$ is a quotient Hopf algebra of the $p$-primary Steenrod algebra $A^{*}$.

Now according to [11] there exist integral lifts of the $\mathscr{P}^{s}$ to operations $\mathscr{P}^{s}$ on $H^{*}\left(B U ; \mathbb{Z}_{(p)}\right)$. These satisfy

$$
\mathscr{P}^{s} s_{k}=\left(\begin{array}{l}
k \\
s
\end{array}\right) s_{k+(p-1) s}
$$


We will make use of the total operations

$$
\mathscr{P}=\sum_{0 \leqq s} \mathscr{P}^{s}, \quad \mathscr{P}=\sum_{0 \leqq s} \not{P} s
$$

which are Hopf algebra homomorphisms; thus

$$
\mathscr{P}(x y)=\mathscr{P}(x) \mathscr{P}(y), \quad \widetilde{P}(x y)=\mathscr{P}(x) \mathscr{P}(y) .
$$

Now to calculate $\mathscr{P}_{a_{k, j}}$ (or more generally $\mathscr{P}_{a_{k, j}}^{(r)}$ ) we can use (1.2) (or (1.5)) and (1.8) recursively. Then $\mathscr{P P}^{s} a_{k, j}\left(\right.$ or $\left.\mathscr{P}^{s} a_{k, j}^{(r)}\right)$ is obtained by reduction $(\bmod p)$.

We can also obtain induced maps on the indecomposable quotients (by multiplicativity)

$$
Q H^{*}(B U ; R)=\tilde{H}^{*}(B U ; R) / \tilde{H}^{*}(B U ; R)^{2}
$$

for $R=\mathbb{Z}_{(p)}$ or $\mathbb{F}_{p}$. We will denote these by $Q \mathscr{P} s^{s}$ and $Q \mathscr{P}^{s}$ respectively.

Of course, everything we have said for $B U$ can be restricted to $W$.

We will use some notation from [9]. Let $\alpha_{p}(k)$ denote the sum $\sum_{i} u_{i}$ where $k=\sum_{i} u_{i} p^{i}$ with $0 \leqq u_{i} \leqq p-1$. Let $M(k)=\left\{t: p^{t}\right.$ divides $\left.k\right\}$. Let

$$
L(k)=\frac{\alpha_{p}(k(p-1))}{(p-1)}
$$

(note that since in general

$$
M(n !)=\frac{\left(n-\alpha_{p}(n)\right)}{(p-1)}
$$

when $n=k(p-1)$, we see that $L(k)$ is an integer!).

Our most important technical result in this section is the following.

Lemma 1.9. Consider the restriction

$$
Q \mathscr{P P s}^{s} Q \bar{B}[2(p-1) k] \rightarrow Q H^{*}(W)
$$

with $(k, p)=1$. Suppose $L(k)+r=n+1$.

(a) If $Q \mathscr{P s} a_{k(p-1), r} \neq 0$, then $L\left(k p^{r}+s\right)+M\left(k p^{r}+s\right) \leqq n+1$.

(b) There exists an such that $a_{(p-1) k, r} \in \operatorname{im} Q \mathscr{P} s$.

Proof. We have, upon making the identification $s_{k}=q_{k}$ in $H^{*}(B U ; R)$ and using (1.2), (1.8)

$$
\check{P}^{s} a_{k(p-1), r} \equiv p^{t-r}\left(\begin{array}{c}
k(p-1) p^{r} \\
s
\end{array}\right) a_{m, t} \quad(\bmod \text { decomposables) }
$$


where $t=M\left(k p^{r}+s\right)$, and $m p^{t}=\left(k p^{r}+s\right)$. But now it is easily checked that this becomes congruent $(\bmod p)$ to

$$
\frac{k}{m}\left(\begin{array}{c}
k(p-1) p^{r}-1 \\
s
\end{array}\right) a_{m, t}
$$

But now we can apply the argument used by Kochman in the proof of [9, Theorem 6.2] to deduce our result.

We will also need a related result in the last section:

Lemma 1.10. If $s<(p-1) p^{r}$, then

$$
Q \mathscr{P}^{s} a_{(p-1), r} \neq 0 \text { in } Q H^{*}(W) .
$$

Proof. This reduces (see the last proof) to showing

$$
\left(\begin{array}{c}
(p-1) p^{r}-1 \\
s
\end{array}\right) \equiv 0(\bmod p) .
$$

But we have $(p-1) p^{r}-1=(p-2) p^{r}+(p-1)\left(p^{r-1}+p^{r-2}+\cdots+p+1\right)$

and if $s=\Sigma s_{i} p^{i}$ with $0 \leqq s_{i} \leqq p-2$, then

$$
\left(\begin{array}{c}
(p-1) p^{r}-1 \\
s
\end{array}\right) \equiv\left(\begin{array}{c}
p-2 \\
s_{r}
\end{array}\right)\left(\begin{array}{c}
p-1 \\
s_{r-1}
\end{array}\right) \cdots\left(\begin{array}{c}
p-1 \\
s_{0}
\end{array}\right)(\bmod p)
$$

easily implies the result.

We are now ready to define inductively a family of quotient $\mathscr{P D}^{*}$ Hopf algebra $C(n)^{*}$ of $H^{*}(W)$ and $\mathscr{P}^{*}$ Hopf ideals $J_{n}$ of $C(n)^{*}$.

Set $C(0)^{*}=H^{*}(W), J_{0}=\left\langle\mathscr{P}^{*} a_{p-1,0}\right\rangle$. Now suppose we have defined $C(n)^{*}$ and $J_{n}$. Set

$$
\begin{aligned}
& C(n+1)^{*}=C(n)^{*} / J_{n} \\
& J_{n+1}=\left\langle\mathscr{P}^{*} \bar{a}_{p-1, n+1}\right\rangle .
\end{aligned}
$$

In this definition we use $\bar{x}$ to denote the reduction of $x \in H^{*}(W)$ to $C(n)^{*}$, and $\left\langle\mathscr{P P}^{*} a\right\rangle$ to denote the ideal generated by the $\mathscr{P}^{*}$ module generated by $a \in C(x)^{*}$.

Theorem 1.11. (a) Each $C(n)^{*}$ is a $\mathscr{P}^{*}$ quotient Hopf algebra of $C(n-1)^{*}$ and of $H^{*}(W)$; each $J_{n}$ is a $\mathscr{P P}^{*}$ Hopf ideal of $C(n)^{*}$.

(b) There is a compatible decomposition of $\mathbb{F}_{p}$ Hopf algebras

$$
C(n)^{*}=\bigotimes_{(k, p)=1} \bar{B}[2(p-1) k] / / \mathbb{F}_{p}\left[a_{(p-1) k, j:} \quad 0 \leqq j<\rho_{n}(k)\right]
$$

where $\rho_{n}(k)=\min \left\{t: L\left(k p^{t}\right)+t>n\right\}$.

(c) $C(n)^{*}$ agrees with Kochman's ${ }_{p} B^{*}(n)([9$, Section 6$])$. 
Proof. (a) is straightforward.

(b) Suppose the result holds for $C(n)^{*}$. We need to verify that as $\mathbb{F}_{p}$ vector spaces

$$
Q C(n+1)^{*}=\mathbb{F}_{p}\left\{\bar{a}_{(p-1) k, j}:(k, p)=1, \quad j \geqq \rho_{n+1}(k)\right\}
$$

where $\bar{a}_{[p-1) k, j}$ are non-zero indecomposables. But this is a consequence of Lemma 1.9. We also need to note that the indecomposable elements of $J_{n}$ are primitive, and hence each one is contained in a factor $\bar{B}[2(p-1) k] / / \mathbb{F}_{p}\left[a_{(p-1) k, j}: 0 \leqq j<\rho_{n}(k)\right]$ by a check on the primitives of $C(n)^{*}$.

(c) If we have the result that $C(n)^{*}={ }_{p} B^{*}(n)$, then because ${ }_{p} B^{*}(n)$ is $2(p-1) p^{n}-1$ connected, $\bar{a}_{(p-1), n}$ agrees with $\bar{v}_{p^{n}}$ (up to $\mathbb{F}_{p}$ unit) in $B^{*}(n)$. Thus by definition $C(n+1)^{*}=$ ${ }_{p} B^{*}(n+1)$.

Of course, Kochman's Theorem 6.2 can be applied to our $C(n)^{*}$, and we will sometimes use results stated in his terminology.

Proposition 1.12. $C(n)^{*}$ is a bipolynomial, self dual $\mathbb{F}_{p}-$ Hopf algebra.

Proof. The Verschiebung $V$ iterated $r$ times induces an isomorphism

$$
B[2 k] / / \mathbb{F}_{p}\left[a_{k, j}: 0 \leqq j<r\right] \cong B[2 k] .
$$

We next describe the dual $C(n)_{*}$ of $C(n)^{*}$. Since $C(n)^{*}$ is a quotient $H^{*}(W)$, we will consider $C(n)_{*}$ as canonically embedded in $H_{*}(W)$ as a sub-Hopf algebra. To avoid doubling notation we will also use the self duality of $B[2 k]$, so that for example we have from [8]

$$
H_{*}\left(B U ; \mathbb{Z}_{(p)}\right)=\bigotimes_{(k, p)=1} B[2 k]
$$

where we now regard $B[2 k]$ as a sub-Hopf algebra of $H_{*}\left(B U ; \mathbb{Z}_{(p)}\right)$.

Theorem 1.13. (a) $C(n)_{*}$ has a canonical decomposition as $\mathbb{F}_{p}$ Hopf algebras

$$
C(n)_{*}=\bigotimes_{(k, p)=1} \bar{B}^{\left(\rho_{n}(k)\right)}[2(p-1) k] .
$$

(b) $C(n)_{*}$ is a polynomial algebra on generators $X_{m}(n)$ of degree $2 m(p-1)$ whenever $L(m)+M(m)>n$. Moreover, we can take

$$
X_{m}(n)=a_{k(p-1), r}^{\left(\rho_{n}(k)\right)} \equiv a_{k(p-1), r}^{\rho_{n}(k)}(\bmod p)
$$

(c) The primitives in $C(n)_{*}$ are generated by the elements $q_{m(p-1)} \in H_{2 m(p-1)}(W)$ with $L(m)+M(m)>n$, where $q_{k} \in H_{2 k}(B U)$ is the canonical primitive generator.

Note that we can lift $C(n)_{*}$ to an integral $\mathbb{Z}_{(p)}$-sub-Hopf algebra of $H_{*}\left(W ; \mathbb{Z}_{(p)}\right)$ 


$$
\widetilde{C}(n)_{*}=\bigotimes_{(k, p)=1} B^{\left(\rho_{n}(k)\right)}[2(p-1) k]
$$

The dual of this, $\widetilde{C}(n)^{*}$, is of course a quotient $\widetilde{\mathscr{P} *}$ Hopf algebra of $H^{*}\left(W ; \mathbb{Z}_{(p)}\right)$ in the obvious sense. Notice that everything we have said about $H^{*}(W)$ can be mimicked in the case of a complex oriented theory such as $B P^{*}()$ with $p$-local coefficients, and we can then obtain analogous sub-Hopf algebras of the homology. We hope to return to a "universal" description of the related splittings in later work.

Observe also that the above constructions have analogues for $H^{*}\left(B O ; \mathbb{F}_{2}\right)$, with $\mathscr{P}^{s}$ replaced by $S q^{s}$. This can be obtained directly from what we have described for the case $p=2$ (since then $W=B U$ ) by use of the "squaring" isomorphism. We leave the precise details to the reader.

2.

We will now describe the (homology) action of the algebra of pth power operations $\mathscr{P P}^{*}$ on $C(n)_{*}$, and the coaction

$$
\psi: C(n)_{*} \rightarrow \mathscr{P}_{*} \otimes C(n)_{*}
$$

of the dual $\mathscr{P}_{*}$ of $\mathscr{P P}$.

Recall that $\mathscr{P}_{*}$ is a sub-Hopf algebra of the dual Steenrod algebra $A_{*}$, with

$$
\begin{aligned}
\mathscr{P}_{*} & =\mathbb{F}_{p}\left[\xi_{i}: i \geqq 1\right] \\
& =\mathbb{F}_{p}\left[\zeta_{i}: i \geqq 1\right]
\end{aligned}
$$

where $\left|\xi_{i}\right|=\left|\zeta_{i}\right|=2\left(p^{i}-1\right)$, and $\zeta_{i}=\chi\left(\xi_{i}\right)$ with $\chi$ the canonical conjugation on $\mathscr{P}_{*}$. The coproduct $\psi$ on $\mathscr{P}_{*}$ is given by

$$
\left.\begin{array}{l}
\psi \xi_{i}=\sum_{0 \leqq j \leqq i} \xi_{i-j}^{p^{j}} \otimes \xi_{j} \\
\psi \zeta_{i}=\sum_{0 \leqq j \leqq i} \zeta_{i} \otimes \zeta_{i-j}^{p^{j}}
\end{array}\right\}
$$

We will often make use of a power series (generating function) description of such results. Thus if we write

$$
\begin{aligned}
& \zeta(T)=\sum_{0 \leqq i} \xi_{i} T^{p^{i}} \\
& \zeta(T)=\sum_{0 \leqq i} \zeta_{i} T^{p^{i}}
\end{aligned}
$$

then

$$
\begin{aligned}
& \psi \xi(T)=(1 \otimes \xi) \circ(\xi \otimes 1)(T) \\
& \psi \zeta(T)=(\zeta \otimes 1) \circ(1 \otimes \zeta)(T)
\end{aligned}
$$


where $\circ$ denotes the functional composition of power series. We use the standard conventions for products in the tensor product of two graded algebras, and denote by $1 \otimes \xi, \xi \otimes 1$, etc., the series

$$
\begin{aligned}
& (1 \otimes \xi)(T)=\sum_{i} 1 \otimes \xi_{i} T^{p^{i}} \\
& (\xi \otimes 1)(T)=\sum_{i} \xi_{i} \otimes 1 T^{p^{i}}
\end{aligned}
$$

and so on.

Now let $H_{*}(B U)=\mathbb{F}_{p}\left[b_{i}: i \geqq 1\right]$ where $\left|b_{i}\right|=2 i$, with $b_{i}$ the standard generator coming from $B U(1)[1]$. The diagonal is

$$
\Delta\left(b_{i}\right)=\sum_{0 \leqq j \leqq 1} b_{j} \otimes b_{i-j}
$$

and the primitives are generated by an element $q_{i} \in H_{2 i}(B U)$ given by the Newton polynomial in the $b_{i}$ :

$$
q_{i}=b_{1} q_{i-1}-b_{2} q_{i-2}+\cdots+(-1)^{i-2} b_{i-1} q_{1}+(-1)^{i-1} i b_{i} .
$$

Setting $b(T)=\sum_{0 \leqq i} b_{i} T^{i}$ with $b_{0}=1$, we can give a generating function for the $q_{i}$,

$$
\sum_{1 \leqq j}(-1)^{j} q_{j} T^{j}=-T \frac{b^{\prime}(T)}{b(T)}
$$

(here $b^{\prime}(T)$ denotes the formal derivative).

The coaction $\psi: H_{*}(B U) \rightarrow \mathscr{P}_{*} \otimes H_{*}(B U)$ now takes the form

$$
\psi b(T)=(1 \otimes b) \circ(\xi \otimes 1)(T)
$$

or

$$
\psi b_{i}=\sum_{1 \leqq j \leqq i}\left[\xi(T)^{\dot{j}}\right]_{T^{i}} \otimes b_{j}
$$

where $[f(T)]_{T^{k}}$ denotes the coefficient of $T^{k}$ in $f(T)$. This result can be found in [2] and [17]. We need a formula for the coaction on the $q_{i}$, which is probably well known to the experts, but not easy to find in the literature in this form.

Proposition 2.6. We have

$$
\psi \sum_{1 \leqq j}(-1)^{j} q_{j} T^{j}=\frac{T}{(\xi \otimes 1)(T)}(\xi \otimes 1)^{\prime}(T) \sum_{1 \leqq k}(-1)^{k} \xi(T)^{k} \otimes q_{k} .
$$

Proof. This is just a formal manipulation with the logarithmic derivative. The presence of torsion does not affect the result, since the whole calculation could in fact be 
lifted to an integral version using the methods of [11]; alternatively we could lift back to $B P_{*}()$.

A simplification is actually possible, since

$$
\begin{aligned}
\xi^{\prime}(T) & =\sum_{0 \leqq i} p^{i} \xi_{i} T^{p^{i}-1} \\
& =1 .
\end{aligned}
$$

Hence, (2.6) gives

$$
\psi \sum_{1 \leqq j}(-1)^{j} q_{j} T^{j}=\frac{T}{(\xi \otimes 1)(T)} \sum_{1 \leqq k}(-1)^{k} \xi(T)^{k} \otimes q_{k}
$$

We will need some rather technical results on the coproduct for certain elements of $H_{*}(W)$. Let

$$
m_{k}=\frac{1}{(k+1)}\left[b(T)^{-k-1}\right]_{T *}
$$

Then by Lagrange inversion, the series $\sum_{0 \leqq k} m_{k} T^{k+1}$ is the composition inverse of the series $b(T)=T b(T)$. Of course the above formula for $m_{k}$ means "work in characteristic 0 until the last possible moment!".

Lemma 2.8. $q_{p^{r-1}}=-m_{p^{r-1}}$ in $H_{*}(B U)$.

Proof. Suppose $x_{1}, x_{2}, x_{3}, \ldots$ are formal indeterminates. Then set $b_{i}=\Sigma x_{1} \cdots x_{i}$ (the $i$ th elementary symmetric function), hence

$$
b(T)=\prod_{i}\left(1+x_{i} T\right)
$$

Now we have

$$
\begin{aligned}
b(T)^{-p^{r}} & =\prod_{i}\left(1+x_{i} T\right)^{-p^{\prime}} \\
& =\prod_{i}\left(\sum_{0 \leqq s}\left(\begin{array}{c}
-p^{r} \\
s
\end{array}\right) x_{i}^{s} T^{s}\right) .
\end{aligned}
$$

But

$$
\left(\begin{array}{c}
-p^{r} \\
s
\end{array}\right)=(-1)^{s} \frac{\left(p^{r}+1\right)\left(p^{r}+2\right) \cdots\left(p^{r}+s-1\right) \cdot p^{r}}{1 \cdot 2 \cdots \cdots \cdot(s-1) \cdot s}
$$

and so for $s<p^{r}$, the exponent of $p$ in this coefficient is $r-M_{p}(s)$, and thus always at least 1 . But the coefficient of $T^{r^{\prime}-1}$ in $b(T)^{-p^{\prime}}$ is obtained by summing products (over 
various $i$ ) of such terms and at least one of the factors always an odd value of $s$. Thus, the $p$ exponent is always at least $r+1$, except when only one value of $i$ occurs. Hence, $\left(\bmod p^{r+1}\right)$ we have

$$
\left(\begin{array}{c}
-p^{r} \\
p^{r}-1
\end{array}\right) \Sigma x_{1}^{p^{r}-1}
$$

as the desired coefficient, and this yields

$$
(-1) \Sigma x_{1}^{p^{r}-1}(\bmod p)
$$

Finally, we recall that if $b_{i}=\Sigma x_{1}, \ldots, x_{i}$, then $q_{i}=\Sigma x_{1}^{i}$.

As a consequence we have

\section{Corollary 2.9 .}

$$
\left[\frac{T}{\xi(T)}\right]_{p^{r-1}}=-\zeta_{r}
$$

Proof. By the remark preceding (2.7) we need to calculate with

$$
\frac{T \xi^{\prime}(T)}{\xi(T)}
$$

Recall that there is a commuting diagram of spectra

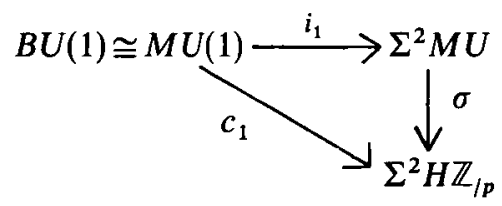

where $i_{1}$ is the map including $M U(1)$ into the second stage of the spectrum $\Sigma^{2} M U, c_{1}$ is the map classifying the universal first Chern class, and $\sigma$ is the universal orientation for complex bundles. If $\left\{b_{i}\right\}$ denotes the standard basis of $H_{*}(B U(1))$, we have

$$
\begin{aligned}
c_{1} * b_{i+1} & =\xi_{r}, \quad \text { if } i+1=p^{r}, \\
& =0, \quad \text { otherwise. }
\end{aligned}
$$

On the other hand, $i_{1} * b_{i+1}$ is identical with $\Phi\left(b_{i}\right)$ where

$$
\Phi: H_{*}(B U) \rightarrow H_{*}(M U)
$$

is the Thom isomorphism induced by $\sigma$, and $H_{*}(B U(1)) \subset H_{*}(B U)$. But now we need 
only quote from [18] the result

$$
\begin{aligned}
& \sigma_{*} \Phi\left(m_{i}\right)=\zeta_{r}, \quad i=p^{r}-1, \\
& =0 \text {, otherwise }
\end{aligned}
$$

and we can safely leave the reader to complete the argument using (2.4) and (2.8) together with the properties of $\sigma$, which is a map of ring spectra.

Notice that in terms of $(1.13 \mathrm{~b}), C(n)_{*}$ has as the first generator of each factor of form

$$
\bar{B}^{\left(\rho_{n}\left(p^{r}-1\right)\right)}\left[2\left(p^{r}-1\right)\right]
$$

a power of $m_{p^{r-1}}$.

We can now give an algorithm for computing $\psi a_{k, j}^{(r)}$.

Algorithm 2.10. First interpret (1.5) and (2.6) as integral formulae (i.e. defined over $\left.H_{*}\left(B U ; \mathbb{Z}_{(p)}\right)\right)$. Treating $\psi$ as a ring homomorphism, use the values of $\psi q_{k p^{r+j}}$ given by (2.8) to recursively determine $\psi q_{k, j}^{(r)}$. Finally, reduce $(\bmod p)$.

This can be justified using the homology coproduct analogue of Lance's integral lifting, or by pulling back to Brown-Peterson homology.

The homology action of $\mathscr{P}^{*}$ on $H_{*}(B U)$ can be computed by similar techniques from the basic integral result of [11]:

$$
\check{\mathscr{P}}_{*} q_{k}=\frac{k}{(k-s(p-1))}\left(\begin{array}{c}
k-s(p-1) \\
s
\end{array}\right) p_{k-s(p-1)}
$$

An independent proof of this can be obtained by calculating the coefficient of $\xi_{1}^{s} \otimes q_{k-s(p-1)}$ in $\psi q_{k}$ as given by (2.6) interpreted integrally rather than $(\bmod p)$.

We have now given all of the necessary formulae for determining the coaction $\psi$ and the actions of the Steenrod powers on the generators $a_{k, j}^{(r)}$ of $H_{*}(B U)$ and hence of each $C(n)_{*}$, by (1.13).

3.

In this section we will describe the corresponding $\mathscr{P}_{*}$ sub-comodule algebras of $H_{*}(M U)$. Recall that there is a multiplicative Thom isomorphism $\Phi: H_{*}(B U ; R) \rightarrow H_{*}(M U ; R)$, and dually a coalgebra isomorphism $\Phi: H^{*}(B U ; R) \rightarrow H^{*}(M U ; R)$. For a prime $p$, we define

$$
M(n)_{*}=\Phi\left(C(n)_{*}\right)
$$

and

$$
M(n)^{*}=\Phi\left(C(n)_{*}\right)^{*}
$$

Then $M(n)_{*}$ is a sub-algebra of $H_{*}(W)$ and $M(n)^{*}$ a quotient coalgebra of $H^{*}(W)$. 
The cohomology Thom isomorphism is given by

$$
\Phi(a)=a \cdot U
$$

for $a \in H^{*}(B U)$, where $U \in H^{0}(M U)$ is the canonical orientation class; the product in this formula is an external pairing of $B U$ with $M U$; then $\Phi$ is by definition an $H^{*}(B U)$ module homomorphism. However, it is not a $\mathscr{P}^{*}$ module homomorphism.

Theorem 3.1. (a) Each $M(n)^{*}$ is a quotient $\mathscr{P}^{*}$ module coalgebra of $M(n-1)^{*}$, and hence of $H^{*}(W)$. Dually, each $M(n)_{*}$ is a $\mathscr{P}_{*}$ sub-module algebra of $M(n-1)_{*}$, hence of $H_{*}(W)$.

(b) $M(n)^{*}$ and $M(n)_{*}$ agree with Kochman's ${ }_{p} M(n)^{*}$ and ${ }_{p} M(n)_{*}([10])$.

(c) There is a coalgebra decomposition

$$
M(n)^{*} \cong \bigotimes_{(k, p)=1} \bar{B}[2(p-1) k] / / \mathbb{F}_{p}\left[a_{(p-1) k, j}: 0 \leqq j<\rho_{n}(k)\right]
$$

and an algebra decomposition

$$
M(n)_{*} \cong \bigotimes_{(k, \rho)=1} \bar{B}^{\left(\rho_{n}(k)\right)}[2(p-1) k]
$$

Note that there is no diagonal map for spectra in general, and hence no geometrically induced product on $H^{*}(M U)$, or coproduct on $H_{*}(M U)$. We can of course define such structures with the aid of $\Phi$ in each case; then these become Hopf algebras and $\Phi$ is a Hopf algebra isomorphism in both cases, and in (3.1c) the isomorphisms are of Hopf algebras-although not over $\mathscr{P}^{*}$ or $\mathscr{P}_{*}$.

To avoid unnecessarily elaborate notation we will consider $\Phi$ as identifying $C(n)^{*}$ with $M(n)^{*}$ and $C(n)_{*}$ with $M(n)_{*}$; thus we can use the notation already established for $C(n)$ to label elements of $M(n)_{*}$. With this convention we can regard $M(n)$ as defining new $\mathscr{P P}^{*}$ module (or $\mathscr{P}_{*}$ comodule) structures on $C(n)^{*}$ (or $C(n)_{*}$ ).

Next recall that for each $n$, there is a sub-Hopf algebra of $\mathscr{P}^{*}$, generated by the $\mathscr{P}^{s}$, $1 \leqq s \leqq p^{n-1}$. We will denote this by $\mathscr{P}(n)^{*}$. Dually, there is a quotient Hopf algebra of $\mathscr{P}_{*}$,

$$
\begin{aligned}
\mathscr{P}(n)_{*} & =\mathscr{P}_{*} / / \mathbb{F}_{p}\left[\xi_{1}^{p^{n}}, \xi_{2}^{p^{n-1}}, \ldots, \xi_{n}^{p}, \xi_{n+1}, \xi_{n+2}, \ldots\right] \\
& =\mathscr{P}_{*} / / \mathbb{F}_{p}\left[\zeta_{1}^{p^{n}}, \zeta_{2}^{n^{n-1}}, \ldots, \zeta_{n}^{p}, \zeta_{n+1}, \zeta_{n+2}, \ldots\right]
\end{aligned}
$$

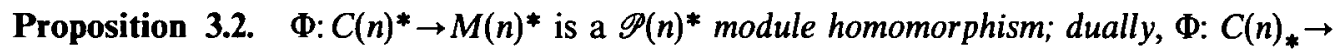
$M(n)_{*}$ is a $\mathscr{P}(n)_{*}$ comodule homomorphism.

Proof. $C(n)^{*}$ is $2(p-1) p^{n}-1$ connected by $(1.11)$, hence for $0<s \leqq p^{n-1}, \mathscr{P}^{s} U=0$ in $M(n)^{*}$. But then for $0<s \leqq p^{n-1}$, 
DECOMPOSITIONS AND ACTIONS OF THE STEENROD ALGEBRA 283

$$
\begin{aligned}
\Phi\left(\mathscr{P}^{s} a\right) & =\left(\mathscr{P P}^{s} a\right) \cdot U \\
& =\sum_{0 \leqq i \leqq s}\left(\mathscr{P}^{i} a\right) \cdot\left(\mathscr{P}^{s-i} U\right) \\
& =\mathscr{P}^{s}(a \cdot U) \text { (by the Cartan formula) } \\
& =\mathscr{P}^{s}(\Phi(a)) .
\end{aligned}
$$

Thus, the $\mathscr{P}(n)^{*}$ structure of $M(n)^{*}$ is determined by that $C(n)^{*}$. Indeed we have

Theorem $3.3([15,10])$. Let $I_{n}=\left\langle q_{p^{r}-1}^{\rho_{n}\left(p^{r}-1\right)}: r \geqq 1\right\rangle$ be the ideal in $M(n)_{*}$ generated by the bottom generators of the factors of form $\bar{B}^{\left(\rho_{n}\left(p^{r}-1\right)\right)}\left[2\left(p^{r}-1\right)\right]$ in $(3.1 c)$.

Then $I_{n}$ is a $\mathscr{P}(n)_{*}$ sub-comodule of $M(n)_{*}$, and there is an isomorphism of left $\mathscr{P}_{*}$ comodule algebras

$$
M(n)_{*} \cong \mathscr{P}_{*} \square_{\mathscr{P}(n) *}\left(M(n)_{*} / I_{n}\right)
$$

Furthermore, as algebras

$$
M(n)_{*} / I_{n} \cong \bigotimes_{(k, p)=1} \bar{B}^{\left(\rho_{n}(k)\right)}[2(p-1) k] / I_{n}(k)
$$

where

$$
\begin{aligned}
I_{n}(k) & =0, \quad \text { if }(p-1) k \text { is not of form } p^{r}-1, \\
& =\left\langle q_{p^{r}-1}^{\rho_{n}\left(p^{\prime}-1\right)}\right\rangle \quad \text { if }(p-1) k=p^{r}-1 .
\end{aligned}
$$

In the above, $M(n)$ and $M(n)_{*} / I_{n}$ have the obvious left $\mathscr{P}(n)_{*}$ comodule structures, inherited from their $\mathscr{P}_{*}$ structures; $\mathscr{P}_{*}$ similarly becomes a right $\mathscr{P}(n)_{*}$ comodule. Thus, the cotensor product is defined.

The determination of the $\mathscr{P}(n)_{*}$ structure of $M(n)_{*} / I_{n}$ is the main motivation for this paper. We have now established all the basic ingredients for an investigation of this, including the provision of canonical generators for $M(n)_{*} / I_{n}$, for which the $\mathscr{P}(n)_{*}$ coaction can in principle be calculated. For example, the calculations of [6] can be redone with explicit choices of generators using our approach. We hope that our techniques will prove useful in the so far unsolved case of the $A_{2}$ structure of $H^{*}(M O\langle 8\rangle)$ which is currently under investigation.

We end this section by briefly stating the main results needed to compute the $\mathscr{P}_{*}$ coaction on $M(n)_{*}$.

Recall that

$$
H_{*}(M U)=\mathbb{F}_{p}\left[b_{i}: i \geqq 1\right]
$$

(with our convention about $\Phi$ !) Then let 


$$
\begin{gathered}
\bar{b}(T)=\sum_{0 \leqq i} b_{i} T^{i+1} . \\
\psi \overline{b(T)}=(1 \otimes b) \circ(\xi \otimes 1)(T) \\
\psi \bar{b}_{i}=\sum_{0 \leqq j \leqq i}\left[\xi(T)^{j+1}\right]_{T^{i}} \otimes \bar{b}_{j}
\end{gathered}
$$

(see [17]).

Proposition 3.5.

$$
\psi \sum_{1 \leqq i}(-1)^{i} q_{i} T^{i}=\frac{T(\xi \otimes 1)^{\prime}(T)}{(\xi \otimes 1)(T)}\left[\sum_{1 \leqq j}(-1)^{j} \xi(T)^{j} \otimes q_{j}\right] .
$$

The proof of these uses techniques analogous to those of Section 2; note that (3.3) can be interpreted as an integral formula in the sense of $[11] ;(\bmod p)$ there is the usual simplification resulting from $\xi^{\prime}(T)=1$. We record also the useful result:

Proposition $3.6([5,13])$.

$$
\psi q_{p^{r-1}}=\sum_{0 \leqq i \leqq r} \zeta i \otimes q_{p}^{p_{r-i}^{i}-1}
$$

(N.B. $\left.q_{p^{r-1}}=a_{p^{r-1,0}}\right)$.

4.

In this section we give some results on Hopf algebra endomorphisms which are easily obtained in our current framework. We suspect that these are known to some experts (in various guises) but can only find [14] in the literature. Once again we fix a prime $p$ throughout.

Let $B=\bar{B}[2]$, and write $a_{j}=a_{1, j}, j \geqq 0$. We wish to compute the graded endomorphisms of $B$. Let $u$ be an indeterminate of degree 2. Then we can consider the set of all graded $\mathbb{F}_{p}$ algebra homomorphisms from $B$ to $\mathbb{F}_{p}[u]$,

$$
\operatorname{Alg}_{\mathbb{F}_{p}}\left(B, \mathbb{F}_{p}[u]\right)
$$

This is an abelian group with product induced from the diagonal $\Delta$ on $B$, i.e. the composite

$$
\operatorname{Alg}_{F_{p}}\left(B, \mathbb{F}_{p}[u]\right) \times \operatorname{Alg}_{F_{p}}\left(B, \mathbb{F}_{p}[u]\right) \cong \operatorname{Alg}_{F_{p}}\left(B \otimes B, \mathbb{F}_{p}[u]\right) \stackrel{\Delta^{*}}{\longrightarrow} \operatorname{Alg}_{F_{p}}\left(B, \mathbb{F}_{p}[u]\right)
$$

Actually, the functor on the category of graded $\mathbb{F}_{p}$ algebras

$$
H \leadsto \operatorname{Alg}_{\mathbf{F}_{2}}(B, H)
$$


is an "abelian group scheme" with an obvious generalisation of the above product.

Lemma 4.1. As a group

$$
\left.\operatorname{Alg}_{\mathrm{F}_{p}}\left(B, \mathbb{F}_{p}[u]\right) \cong \mathbb{Z}_{p} \quad \text { (the p-adic integers }\right) .
$$

Proof. Recall that $\mathbb{Z}_{p}$ has maximal ideal $(p)$, and residue field $\mathbb{F}_{p}$. Furthermore, there is a unique subgroup $U^{\times} \subset \mathbb{Z}_{p}^{\times}$for which the canonical projection $\mathbb{Z}_{p} \rightarrow \mathbb{F}_{p}$ induces an isomorphism $U^{\times} \cong \mathbb{F}_{p}^{\times}$. Let $U=U^{\times} \cup\{0\}$, identified with $\mathbb{F}_{p}$.

Then for any $p$-adic integer $c$, there is a unique expansion

$$
c=\sum_{0 \leqq i} c_{i} p^{i}
$$

where $c_{i} \in U$. The sequence $c_{i}$ is called the Teichmüller representation of $c$. Then the essence of the Witt vector addition is that for

$$
\begin{aligned}
& a=\sum_{0 \leqq i} a_{i} p^{i} \quad \text { and } \quad b=\sum_{0 \leqq i} b_{i} p^{i} \quad \text { with } a_{i}, b_{i} \in U, \\
& a+b=\sum_{0 \leqq i} \Sigma_{i}^{W}\left(a_{0}, \ldots, a_{i} ; b_{0}, \ldots, b_{i}\right) p^{i} \quad \text { (see Section 1). }
\end{aligned}
$$

Now define

$$
\Omega: \operatorname{Alg}_{\mathrm{F}_{p}}\left(B, \mathbb{F}_{p}[u]\right) \rightarrow \mathbb{Z}_{p}
$$

by $\Omega(\theta)=\sum_{0 \leqq i} \theta_{i} p^{i}$ where $\theta_{i}$ is a lift to $U$ of the coefficient of $u^{p^{i}}$ in $\theta\left(a_{i}\right)$. Then $\Omega$ is clearly an isomorphism of groups. It can actually be considered as an isomorphism of topological groups if we define a suitable profinite topology on the domain.

Now we observe that any Hopf algebra endomorphism of $B$ induces an endomorphism of the (topological) group $\mathbb{Z}_{p}$, and these are in one to one correspondence with the elements of $\mathbb{Z}_{p}$. Hence, it is now easy to deduce.

Proposition 4.2. $\mathrm{Ho}_{-} \operatorname{Alg}_{\mathrm{F}_{p}}(B, B) \cong \mathbb{Z}_{p}$.

In this description, a positive rational integer $d$ corresponds to an endomorphism [d] given by

$$
B \stackrel{\Psi^{(d)}}{\longrightarrow} B \otimes \cdots \otimes B \stackrel{\phi^{(d)}}{\longrightarrow} B
$$

where there are $d$ copies of $B, \psi^{(d)}$ and $\phi^{(d)}$ are the $d$-fold iterates of the coproduct and product on $B \cdot[-1]$ means (canonical) conjugation. More generally, for a $p$-adic integer $c,[c]$ can be obtained as the limit of a sequence of $[d]$, for $d$ positive rational integers. By (1.4), we have 


$$
\begin{aligned}
{[p]\left(a_{j}\right) } & =0, & & j=0 \\
& =a_{j-1}^{p}, & & j>0 .
\end{aligned}
$$

Note that elements of $\mathbb{Z}_{p}^{\times}$correspond to invertible endomorphisms (automorphisms). We could have described the composition of endomorphisms in terms of Witt multiplication, but refrain from this here.

We now want to describe the $\mathscr{P}^{*}$ Hopf algebra endomorphisms of $C(n)^{*}$.

Note that in (4.1) we can replace $B$ by any $\bar{B}[2 k]$.

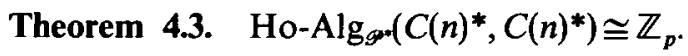

Proof. We will content ourselves with proving the case $n=0$, the others being similar.

For any $\mathscr{P}^{*}$ Hopf algebra endomorphism $\Psi$ of $C(0)^{*}$ we have

(a) $\Psi(\bar{B}[2(p-1) k]) \subset \bar{B}[2(p-1) k]$ for each $(k, p)=1$.

(b) $\Psi$ restricted to $\bar{B}[2(p-1)]$ is $[c]$ for some $c \in \mathbb{Z}_{p}$.

(a) is true for any $\mathbb{F}_{p}$ Hopf algebra endomorphism, by an easy induction and (b) then follows with the aid of (4.2).

We must now link up the $\bar{B}[2(p-1) k]$ to $\bar{B}[2(p-1)]$ by Steenrod operations. To do this recall (1.10):

For

$$
\begin{gathered}
s<(p-1) p^{n}, \\
\mathscr{P s} a_{(p-1), n} \equiv 0 \quad(\bmod \text { decomposables })
\end{gathered}
$$

in $H^{*}(W)$.

We will now prove by induction that if $\Psi$ is $[0]$ on $B[2(p-1)]$, then $\Psi$ is [0] on each $B[2(p-1) k]$, hence is the trivial endomorphism

$$
[0]\left(a_{(p-1) k, j}\right)=0, \quad \text { for } \quad(k, p)=1, \quad j \geqq 0
$$

on $H^{*}(W)$. Thus, if $\Psi$ is any such endomorphism, it is determined by its restriction to $B[2(p-1)]$, by a standard argument in the group of all such endomorphisms.

So suppose we have shown that for $\Psi$ restricting to $[0]$ on $B[2(p-1)]$ we have that $\Psi$ is $[0]$ on

$$
\mathbb{F}_{p}\left[a_{(p-1) k, j}:(k, p)=1, k p^{j}<n\right]
$$

Then choose $m=\max \left\{t: p^{t} \leqq n\right\}$. By (1.10) we have

$$
\mathscr{P}^{n-p^{m}} a_{(p-1), m} \neq 0
$$

in $Q H^{2(p-1) n}(W)$; hence, since 


$$
\Psi\left(\mathscr{P}^{n-p^{m}} a_{(p-1), m}\right)=\mathscr{P}^{n-p^{m}} \Psi\left(a_{(p-1), m}\right)
$$

we have now demonstrated that $\Psi$ agrees with [0] on

$$
\mathbb{F}_{p}\left[a_{(p-1) k, j}:(k, p)=1, k p^{j}<n+1\right] .
$$

This completes the proof of (4.3).

Notice that for $d$ a positive rational integer, we can realise $[d]$ on $H^{*}(W)$ by using the self map of $B U$ classifying $d$ times the canonical bundle on $B U$. If we complete $W$ at $p$, then $[c]$ can be realised for all $c \in \mathbb{Z}_{p}$. Thus, all Steenrod preserving Hopf algebra endomorphisms are geometrically realisable (cf. [14]).

\section{Concluding remarks}

In a planned companion paper we intend to discuss some examples of non-self dual Hopf algebras whose structure can be elucidated with the aid of the Husemoller splittings of $B U$, and which are important in Topology. We also intend to extend our ordinary homology results to theories such as Brown-Peterson homology, by giving an abstract description of the homology of $B U$ as a universal object in a suitable sense. One reason for developing such a point of view is that it should embody the tensor product operation as well as Whitney sum-this is necessary for analysing various problems which we hope to investigate.

Note that our decompositions for $C(n)_{*}$ can be used to calculate the restriction of the actions of the Dyer-Lashof algebra, using results in [11]; we leave the details to the reader.

\section{REFERENCES}

1. F. AdAMs, Lectures on generalised cohology, SLNM 99 (1969), 1-138.

2. F. Adams, Stable Homotopy and Generalised Homology (Math. Lect. Notes, Univ. of Chicago, 1971).

3. A. BAKER, A decomposition theorem for certain bipolynomial Hopf algebras, Can. Math. Bull. 27 (1984).

4. A. Baker, More homology generators for BSU and BSO, Current Trends in Algebraic Topology (Can. Math. Soc. Conf. Proc., 1982), 429-435.

5. E. H. Brown and F. P. Peterson, $H^{*}(M O)$ as an algebra over the Steenrod algebra, Proc. of a Conf. on Homotopy Theory (Soc. Mat. Mex., 1975), 11-19.

6. V. Giambalvo and D. J. Pengelley, The homology of MSpin, Math. Proc. Camb. Phil. Soc. 95 (1984), 427-436.

7. M. Hazewinkel, Formal Groups and Applications (Academic Press, 1978).

8. D. Husemoller, The structure of the Hopf algebra $H_{*}(B U)$ over a $Z_{(p)}$-algebra, Amer. $J$. Math. 93 (1971), 329-349.

9. S. O. Коснмan, An algebraic filtration of $H_{*}(B O)$, Contemp. Math. 19 (1983), 115-143. 
10. S. O. Kochman, An algebraic filtration of $H_{*}(M O)$, preprint.

11. T. Lance, Steenrod and Dyer-Lashof operations on BU, Trans. Am. Math. Soc. 276 (1983), 497-510.

12. J. W. Milnor, The Steenrod algebra and its dual, Ann. of Math. 67 (1958), 150-171.

13. S. A. Mitchell, Power series methods in unoriented cobordism, Contemp. Math. 19 (1983), 247-254.

14. R. Patterson, The square preserving endomorphisms of $H^{*}\left(B O ; Z_{2}\right)$, preprint.

15. D. J. Pengelley, The $A$-structure of Thom spectra: $M S O$ as an example, Current Trends in Algebraic Topology (Can. Math. Soc. Conf. Proc., 1982), 511-513.

16. D. C. Ravenel and W. S. Wilson, Bipolynomial Hopf algebras, J. Pure and App. Alg. 4 (1974), 41-45.

17. R. M. SwITzER, Homology comodules, Invent. Math. 20 (1973), 97-102.

18. R. ZaHLER, The Adams-Novikov spectral sequence for the spheres, Ann. of Math. 96 (1972), 480-504.

Department of Mathematics

ManChester UNIVERSITY

MANChester M13 9PL 